Acta Cryst. (1996). C52, 1449-1451

\section{Cerium 3,5-Dinitrobenzoate Dihydrate}

\author{
M. NAwaz TAhIR, ${ }^{a *}$ Dinçer Üllü̈, ${ }^{a}$ CANan \\ ÜNALEROḠLU $^{b}$ AND ELMAN M. MővsüMOV ${ }^{b}$ \\ ${ }^{a}$ Department of Physics Engineering, Hacettepe University, \\ Beytepe 06532, Ankara, Turkey, and ${ }^{b}$ Department of \\ Chemistry, Hacettepe University, Beytepe 06532, Ankara, \\ Turkey.E-mail: ftahir@eti.cc.hun.edu.tr
}

(Received 14 March 1995; accepted 2 January 1996)

Each $\mathrm{Ce}$ ion is coordinated to six carboxylate $\mathrm{O}$ atoms $(\mathrm{O} 1, \mathrm{O} 2, \mathrm{O} 3, \mathrm{O} 4, \mathrm{O}, \mathrm{O} 6)$ and two water $\mathrm{O}$ atoms (O7, O8) (Fig. 1). The nitro groups are not coordinated to the $\mathrm{Ce}$ ion. The $\mathrm{Ce}-\mathrm{O}$ distances of the bridging carboxylate groups $[2.400(5)-2.507(5) \AA]$ are shorter than the $\mathrm{Ce}-\mathrm{O}$ distances [2.603 (6)-2.661 (5) $\AA$ ] involving the water molecules. A comparison of (I) with known related compounds shows that the average $\mathrm{Ce}-\mathrm{O}$ distance found here $(2.499 \AA)$ is slightly shorter than the value reported for ceric ammonium nitrate [2.508 (7) $\AA$; Beineke \& Delgaudio, 1968], but larger than that found in both $\left[\mathrm{Ce}\left(\mathrm{IO}_{3}\right)_{4}\right]$ [2.31 (9) $\AA$; Cromer \& Allen, 1956] and cerium acetylacetonate [2.395 $\AA$; Matković \& Gredenić, 1963].

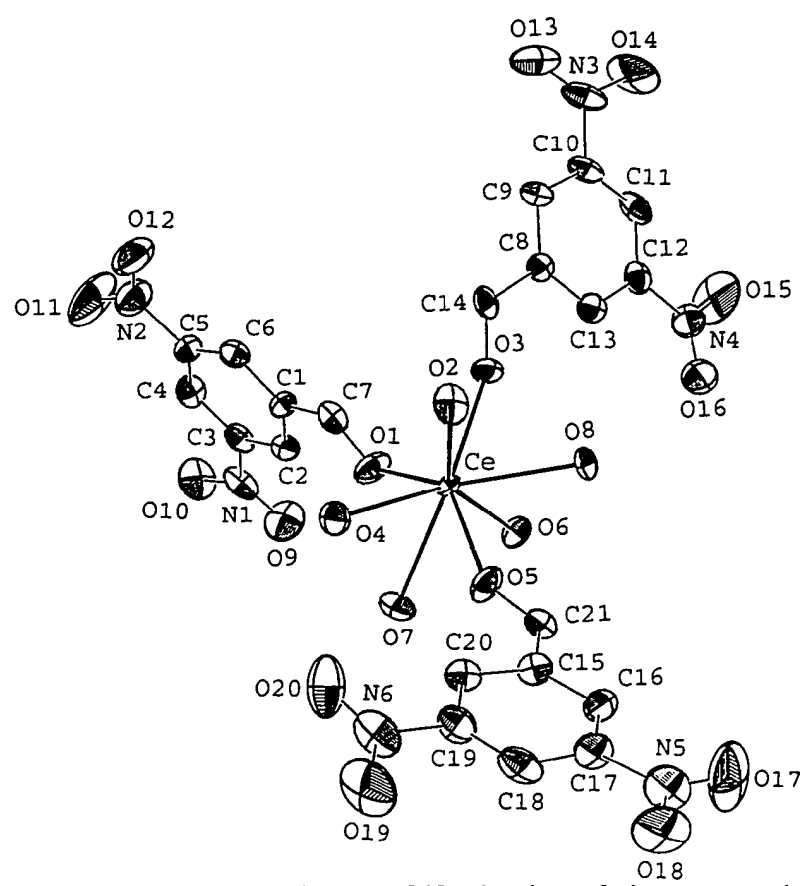

Fig. 1. An ORTEP (Johnson, 1965) drawing of the asymmetric unit of the title compound with the atom-numbering scheme. The displacement ellpsoids are drawn at the $50 \%$ probability level and $\mathrm{H}$ atoms have been omitted for clarity.

the single-mode coordination might be the simultaneous occurrence of two nitro groups. As can be seen from the chemical diagram, $\mathrm{Ce}$ ions are bridged alternately by four and two ligands, resulting in two different $\mathrm{Ce}$... Ce distances of $4.2824(7)$ and $5.3786(8) \AA$, respectively.

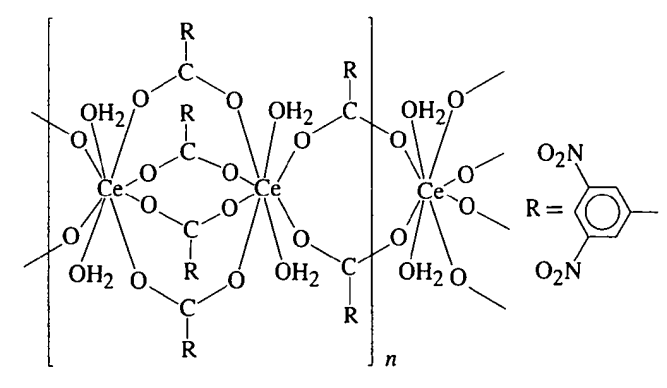

(I)
The average $\mathrm{C}-\mathrm{C}$ distance in the aromatic ring of the 3,5-dinitrobenzoate ligand is 1.38 (1) $\AA$ and the average $\mathrm{C}-\mathrm{N}, \mathrm{C}-\mathrm{O}$ and $\mathrm{N}-\mathrm{O}$ distances are $1.48(1), 1.25(1)$ and $1.214(6) \AA$, respectively. Details of the hydrogenbonding geometry involving the water molecules are given in Table 3.

\section{Experimental}

An aqueous solution of sodium 3,5-dinitrobenzoate was added to an aqueous solution of $\mathrm{CeCl}_{3} .6 \mathrm{H}_{2} \mathrm{O}(1: 1$ molar ratio) and single crystals were obtained by slow evaporation of the final solution. 
Crystal data

$\left[\mathrm{Ce}_{2}\left(\mathrm{C}_{7} \mathrm{H}_{3} \mathrm{~N}_{2} \mathrm{O}_{6}\right)_{6}\left(\mathrm{H}_{2} \mathrm{O}\right)_{4}\right]$

$M_{r}=1618.97$

Triclinic

$P \overline{1}$

$a=9.531$ (1) $\AA$

$b=12.120(1) \AA$

$c=14.327(2) \AA$

$\alpha=64.34(2)^{\circ}$

$\beta=70.61(1)^{\circ}$

$\gamma=82.90(1)^{\circ}$

$V=1406.9(7) \AA^{3}$

$Z=1$

$D_{x}=1.91 \mathrm{Mg} \mathrm{m}^{-3}$

$D_{m}$ not measured

Data collection

Enraf-Nonius CAD-4 diffractometer

$\omega / 2 \theta$ scans

Absorption correction: $\psi$ scans (MolEN; Fair, 1990)

$T_{\min }=0.788, \quad T_{\max }=$ 0.999

5798 measured reflections

5118 independent reflections

\section{Refinement}

Refinement on $F$

$R=0.044$

$w R=0.044$

$S=1.08$

3264 reflections

433 parameters

$w=1 / \sigma^{2}(F)$

$(\Delta / \sigma)_{\max }=0.00031$
Mo $K \alpha$ radiation

$\lambda=0.71073 \AA$

Cell parameters from 25 refiections

$\theta=8.44-20.08^{\circ}$

$\mu=1.73 \mathrm{~mm}^{-1}$

$T=295 \mathrm{~K}$

Needle

$0.25 \times 0.125 \times 0.1 \mathrm{~mm}$

Colourless

3492 observed reflections

$$
[I>3 \sigma(I)]
$$

$R_{\text {int }}=0.030$

$\theta_{\max }=26.32^{\circ}$

$h=0 \rightarrow 11$

$k=-14 \rightarrow 15$

$l=-16 \rightarrow 17$

3 standard reflections frequency: $120 \mathrm{~min}$ intensity decay: $0.85 \%$

$\Delta \rho_{\max }=1.365{\mathrm{e} \AA^{-3}}^{-3}$

$\Delta \rho_{\min }=-0.803 \mathrm{e} \AA^{-3}$

Extinction correction: none

Atomic scattering factors

from International Tables

for X-ray Crystallography (1974, Vol. IV)

Table 1. Fractional atomic coordinates and equivalent isotropic displacement parameters $\left(\AA^{2}\right)$

$$
B_{\mathrm{eq}}=\left(8 \pi^{2} / 3\right) \Sigma_{i} \Sigma_{j} U_{i j} a_{i}^{*} a_{j}^{*} \mathbf{a}_{i} \cdot \mathbf{a}_{j} .
$$

\begin{tabular}{llrrr} 
& \multicolumn{1}{c}{$x$} & \multicolumn{1}{c}{$y$} & \multicolumn{1}{c}{$z$} & $B_{\mathrm{eq}}$ \\
$\mathrm{Ce}$ & $0.77017(5)$ & \multicolumn{1}{c}{$0.02652(4)$} & \multicolumn{1}{c}{$0.00962(3)$} & $1.362(8)$ \\
$\mathrm{O} 1$ & $0.8595(6)$ & $0.2421(5)$ & $-0.0592(4)$ & $3.3(1)$ \\
$\mathrm{O} 2$ & $0.9265(6)$ & $-0.1538(4)$ & $0.0473(4)$ & $3.0(1)$ \\
$\mathrm{O} 3$ & $0.9538(5)$ & $0.0582(5)$ & $-0.1637(3)$ & $2.6(1)$ \\
O4 & $0.8055(6)$ & $-0.0079(5)$ & $0.1835(4)$ & $2.9(1)$ \\
O5 & $0.5659(6)$ & $-0.1100(5)$ & $0.1480(4)$ & $3.0(1)$ \\
O6 & $0.6102(5)$ & $0.1458(4)$ & $-0.0951(3)$ & $2.3(1)$ \\
O7 & $0.5747(6)$ & $0.1554(4)$ & $0.1017(4)$ & $3.0(1)$ \\
O8 & $0.7028(6)$ & $-0.1008(4)$ & $-0.0771(4)$ & $3.0(1)$ \\
O9 & $0.8125(7)$ & $0.6791(5)$ & $-0.1663(5)$ & $4.7(2)$ \\
O10 & $0.9846(7)$ & $0.7838(5)$ & $-0.1695(5)$ & $4.5(2)$ \\
O11 & $1.4459(8)$ & $0.5947(6)$ & $-0.1248(6)$ & $8.1(2)$ \\
O12 & $1.4819(7)$ & $0.4075(6)$ & $-0.1008(5)$ & $5.4(2)$ \\
O13 & $1.5196(7)$ & $0.1237(6)$ & $-0.5710(5)$ & $5.0(2)$ \\
O14 & $1.4137(9)$ & $0.1387(7)$ & $-0.6874(4)$ & $7.3(2)$ \\
O15 & $0.8895(8)$ & $0.2123(8)$ & $-0.6144(5)$ & $7.1(2)$ \\
O16 & $0.7522(7)$ & $0.1227(6)$ & $-0.4480(4)$ & $4.6(2)$ \\
O17 & $0.0933(9)$ & $-0.5259(7)$ & $0.3363(5)$ & $8.8(2)$ \\
O18 & $0.0860(8)$ & $-0.6173(5)$ & $0.5033(5)$ & $5.6(2)$ \\
O19 & $0.3639(9)$ & $-0.4554(7)$ & $0.6422(5)$ & $6.4(2)$ \\
O20 & $0.5454(7)$ & $-0.3294(6)$ & $0.5281(4)$ & $5.1(2)$ \\
N1 & $0.9357(8)$ & $0.6902(5)$ & $-0.1621(4)$ & $3.1(2)$ \\
N2 & $1.4076(8)$ & $0.4990(6)$ & $-0.1157(6)$ & $4.2(2)$
\end{tabular}

$\begin{array}{llrrr}\text { N3 } & 1.4096(8) & 0.1288(6) & -0.5974(5) & 3.5(2) \\ \text { N4 } & 0.8710(8) & 0.1601(6) & -0.5181(5) & 3.5(2) \\ \text { N5 } & 0.1304(9) & -0.5361(6) & 0.4120(6) & 4.7(2) \\ \text { N6 } & 0.4332(8) & -0.3846(6) & 0.5501(5) & 3.8(2) \\ \text { C1 } & 1.0604(8) & 0.3691(6) & -0.0985(5) & 1.8(2) \\ \text { C2 } & 0.9726(8) & 0.4742(6) & -0.1223(5) & 2.1(2) \\ \text { C3 } & 1.0332(9) & 0.5824(6) & -0.1441(5) & 2.4(2) \\ \text { C4 } & 1.1735(9) & 0.5945(7) & -0.1439(5) & 2.8(2) \\ \text { C5 } & 1.2579(8) & 0.4911(7) & -0.1211(5) & 2.6(2) \\ \text { C6 } & 1.2041(8) & 0.3777(6) & -0.0997(5) & 2.3(2) \\ \text { C7 } & 0.9931(9) & 0.2455(6) & -0.0662(5) & 2.3(2) \\ \text { C8 } & 1.1058(8) & 0.0770(6) & -0.3369(5) & 1.9(2) \\ \text { C9 } & 1.2499(9) & 0.0856(6) & -0.4105(5) & 2.3(2) \\ \text { C10 } & 1.2601(9) & 0.1202(7) & -0.5173(5) & 2.6(2) \\ \text { C11 } & 1.1417(9) & 0.1447(7) & -0.5564(5) & 2.8(2) \\ \text { C12 } & 1.0023(9) & 0.1320(6) & -0.4799(5) & 2.5(2) \\ \text { C13 } & 0.9835(9) & 0.1004(6) & -0.3727(5) & 2.4(2) \\ \text { C14 } & 1.0834(8) & 0.0450(6) & -0.2185(5) & 2.2(2) \\ \text { C15 } & 0.3933(8) & -0.2695(6) & 0.2741(5) & 2.3(2) \\ \text { C16 } & 0.2879(8) & -0.3524(7) & 0.2926(6) & 2.6(2) \\ \text { C17 } & 0.2352(9) & -0.4432(7) & 0.3944(6) & 3.0(2) \\ \text { C18 } & 0.275(1) & -0.4542(7) & 0.4823(6) & 3.4(2) \\ \text { C19 } & 0.3817(9) & -0.3725(7) & 0.4602(6) & 2.9(2) \\ \text { C20 } & 0.4448(9) & -0.2822(6) & 0.3585(5) & 2.4(2) \\ \text { C21 } & 0.4547(8) & -0.1676(6) & 0.1640(5) & 2.1(2)\end{array}$

Table 2. Selected geometric parameters $\left(\AA{ }^{\circ}\right)$

$\begin{array}{lrlr}\mathrm{Ce}-\mathrm{O} 1 & 2.507(5) & \mathrm{Ce}-\mathrm{O} 5 & 2.419(4) \\ \mathrm{Ce}-\mathrm{O} 2 & 2.448(5) & \mathrm{Ce}-\mathrm{O} 6 & 2.400(5) \\ \mathrm{Ce}-\mathrm{O} 3 & 2.415(4) & \mathrm{Ce}-\mathrm{O} 7 & 2.661(5) \\ \mathrm{Ce}-\mathrm{O} 4 & 2.473(6) & \mathrm{Ce}-\mathrm{O} 8 & 2.603(6) \\ \mathrm{O} 1-\mathrm{Ce}-\mathrm{O} 2 & 124.9(2) & \mathrm{O} 3-\mathrm{Ce}-\mathrm{O} 7 & 138.9(1) \\ \mathrm{O} 1-\mathrm{Ce}-\mathrm{O} 3 & 77.6(2) & \mathrm{O} 3-\mathrm{Ce}-\mathrm{O} 8 & 68.9(2) \\ \mathrm{O} 1-\mathrm{Ce}-\mathrm{O} 4 & 86.3(2) & \mathrm{O} 4-\mathrm{Ce}-\mathrm{O} 5 & 75.7(2) \\ \mathrm{O} 1-\mathrm{Ce}-\mathrm{O} 5 & 139.2(2) & \mathrm{O} 4-\mathrm{Ce}-\mathrm{O} 6 & 136.6(2) \\ \mathrm{O} 1-\mathrm{Ce}-\mathrm{O} 6 & 76.8(2) & \mathrm{O} 4-\mathrm{Ce}-\mathrm{O} 7 & 70.3(2) \\ \mathrm{O} 1-\mathrm{Ce}-\mathrm{O} 7 & 67.4(2) & \mathrm{O} 4-\mathrm{Ce}-\mathrm{O} 8 & 138.4(2) \\ \mathrm{O} 1-\mathrm{Ce}-\mathrm{O} 8 & 135.0(2) & \mathrm{O}-\mathrm{Ce}-\mathrm{O} 6 & 91.5(2) \\ \mathrm{O} 2-\mathrm{Ce}-\mathrm{O} 3 & 74.8(2) & \mathrm{O}-\mathrm{Ce}-\mathrm{O} 7 & 72.1(2) \\ \mathrm{O} 2-\mathrm{Ce}-\mathrm{O} 4 & 76.4(2) & \mathrm{O}-\mathrm{Ce}-\mathrm{O} 8 & 73.5(2) \\ \mathrm{O} 2-\mathrm{Ce}-\mathrm{O} 5 & 86.4(2) & \mathrm{O}-\mathrm{Ce}-\mathrm{O} 7 & 66.3(2) \\ \mathrm{O} 2-\mathrm{Ce}-\mathrm{O} 6 & 145.1(2) & \mathrm{O} 6-\mathrm{Ce}-\mathrm{O} 8 & 71.7(2) \\ \mathrm{O} 2-\mathrm{Ce}-\mathrm{O} 7 & 143.7(2) & \mathrm{O} 7-\mathrm{Ce}-\mathrm{O} 8 & 123.8(2) \\ \mathrm{O} 2-\mathrm{Ce}-\mathrm{O} 8 & 74.4(2) & \mathrm{Ce}-\mathrm{O} 1-\mathrm{C} 7 & 111.2(4) \\ \mathrm{O} 3-\mathrm{Ce}-04 & 129.5(2) & \mathrm{Ce}-\mathrm{O} 3-\mathrm{C} 14 & 150.4(5) \\ \mathrm{O} 3-\mathrm{Ce}-\mathrm{O} 5 & 141.2(2) & \mathrm{Ce}-\mathrm{O}-\mathrm{O}-\mathrm{C} 21 & 144.5(5) \\ \mathrm{O} 3-\mathrm{Ce}-\mathrm{O} 6 & 85.7(2) & & \end{array}$

\begin{tabular}{|c|c|c|c|c|}
\hline$D \longrightarrow \mathrm{H} \cdots A$ & $D$ - & $\mathrm{H} \cdots A$ & $D \cdots A$ & $D-\mathrm{H} \cdots A$ \\
\hline $\mathrm{O} 7-\mathrm{H} 71 \cdots \mathrm{O} 12^{\mathrm{i}}$ & 0.96 & 2.59 & $3.449(8)$ & 150 \\
\hline $\mathrm{O} 7-\mathrm{H} 72 \cdots \mathrm{O} 14^{\prime \prime}$ & 0.92 & 1.92 & $2.827(8)$ & 168 \\
\hline $\mathrm{O} 8-\mathrm{H} 81 \cdots \mathrm{O} 10^{\mathrm{in}}$ & 0.94 & 2.26 & $3.039(8)$ & 140 \\
\hline $\mathrm{O} 8-\mathrm{H} 82 \cdots \mathrm{O}^{\mathrm{iv}}$ & 1.02 & 2.11 & $2.968(9)$ & 140 \\
\hline
\end{tabular}

Table 3. Hydrogen-bonding geometry $\left(\AA{ }^{\circ}{ }^{\circ}\right)$

$\mathrm{H}$ atoms bonded to $\mathrm{C}$ atoms were placed geometrically $1.05 \AA$ from their bonded atoms, while the water $\mathrm{H}$ atoms were taken from a difference map. All $\mathrm{H}$-atom displacement parameters were fixed as 1.3 times those of their bonded atoms. A riding model was used for all $\mathrm{H}$ atoms.

Data collection: CAD-4 EXPRESS Software (Enraf-Nonius, 1993). Data reduction: MolEN (Fair, 1990). Program(s) used to solve structure: MolEN SIMPEL. Program(s) used to refine structure: MolEN LSFM. Molecular graphics: MolEN ORTEP (Johnson, 1965). Software used to prepare material for publication: MolEN.

The authors wish to acknowledge the purchase of the CAD-4 diffractometer under grant DPT/TBAGl of the Scientific and Technical Research Council of Turkey. 
Lists of structure factors, anisotropic displacement parameters, $\mathrm{H}$ atom coordinates and complete geometry have been deposited with the IUCr (Reference: FG1076). Copies may be obtained through The Managing Editor, International Union of Crystallography, 5 Abbey Square, Chester CHI 2HU, England.

\section{References}

Bats, J. W., Klaus, R. \& Fuess, H. (1979). Acta Cryst. B35, 12251227.

Beineke, T. A. \& Delgaudio, J. (1968). Inorg. Chem. 7, 715-721. Cromer, D. T. \& Allen, C. L. (1956). Acta Cryst. 9, 1015-1018.
Enraf-Nonius (1993). CAD-4 EXPRESS Software. Version 1.1. EnrafNonius, Delft, The Netherlands.

Fair, C. K. (1990). MolEN. An Interactive Intelligent System for Crystal Structure Analysis. Enraf-Nonius, Delft, The Netherlands.

Hiyalov, M. S., Amiraslanov, I. R., Mamedov, Ch. S. \& Môvsümov, E. M. (1981). J. Struct. Chem. (USSR), T22, 113-119.

Johnson, C. K. (1965). ORTEP. Report ORNL-3794. Oak Ridge National Laboratory, Tennessee, USA.

Ma, J.-F., Jin, Z.-S. \& Ni, J.-Z. (1994a). Acta Cryst. C50, 1008-1010. Ma, J.-F., Jin, Z.-S. \& Ni, J.-Z. (1994b). Acta Cryst. C50, 1010-1012. Matković, B. \& Gredenić, D. (1963). Acta Cryst. 16, 456-461.

Moore, J. W., Glick, M. D. \& Baker, W. A. Jr (1971). J. Am. Chem. Soc. 94, 1858-1865. 